\title{
Incidence of Postpartum Diabetes and Glucose Intolerance among Filipino Patients with Gestational Diabetes Mellitus seen at a Tertiary Hospital
}

\author{
Chandy Lou Malong,,$^{1}$ Aileen Sia-Atanacio, ${ }^{1}$ Aimee Andag-Silva, ${ }^{2}$ Elaine Cunanan ${ }^{1}$ \\ ${ }^{1}$ Section of Endocrinology and Metabolism, Department of Medicine, University of Santo Tomas Hospital, España, Manila, Philippines \\ ${ }^{2}$ Section of Endocrinology and Metabolism, Department of Internal Medicine, De La Salle University Medical Center, Dasmariñas, Cavite, Philippines
}

\begin{abstract}
Objective. Women with gestational diabetes mellitus (GDM) are at increased risk to develop type 2 diabetes. This study aims to determine the incidence of postpartum diabetes and/or glucose intolerance among Filipino GDM patients who delivered at a tertiary hospital in Manila and to compare the risk factors present among these women.

Methodology. 173 Filipino patients were included in this 3-year prospective cohort study. Demographics, clinical variables, and feto-maternal outcomes were recorded. 124 patients returned for follow-up and postpartum glycemic status was determined using $75 \mathrm{~g}$ oral glucose tolerance test.

Results. The incidence of diabetes and prediabetes postpartum was $7.3 \%$ and $34.7 \%$ respectively. Logistic regression analysis showed that multigravid patients $(\mathrm{OR}=2.84 ; 95 \% \mathrm{Cl} 1.20,6.70)$ and those with postpartum obesity $(\mathrm{OR}=2.84$; $95 \% \mathrm{Cl} 1.20,6.70$ ) are more likely to have prediabetes. Diagnosis of GDM at an earlier trimester increases the odds of having postpartum diabetes $(\mathrm{OR}=3.05 ; 95 \% \mathrm{Cl} 1.02,9.18)$. Also, if the body mass index falls under obese class II postpartum, the probability increases 115 times $(95 \%$ ci $3.96,3357.83 ; p=0.006)$.
\end{abstract}

Conclusion. The incidence of postpartum glucose intolerance among Filipino women with GDM is high. Clinicians should be more vigilant and strategies to implement compliance to postpartum glucose testing must be formulated to increase rates of follow-up testing among these women.

Keywords: Gestational diabetes mellitus, postpartum glucose intolerance, postpartum diabetes, incidence, Philippines

\section{INTRODUCTION}

Pregnancy is a diabetogenic state normally associated with a significant $(50 \%)$ decline in insulin sensitivity from the first to third trimester. ${ }^{1}$ This insulin resistance can unmask even the slightest defect in insulin secretion, resulting in glucose intolerance and gestational diabetes mellitus (GDM). ${ }^{2}$ GDM, defined as any degree of glucose intolerance with onset or first recognition during pregnancy, is reported to have a prevalence of $14 \%$ in the Philippines and $7.5 \%$ at the University of Santo Tomas Hospital, a tertiary hospital in Manila. ${ }^{3,4}$ The majority of these women appear to have beta cell dysfunction. Thus, the physiologic decrease in insulin sensitivity that occurs during pregnancy appears to be additive to this state of chronic insulin resistance. The compensatory increase in insulin secretion may be inadequate because of beta cell dysfunction resulting in hyperglycemia which may persist beyond pregnancy. ${ }^{5,6}$ Although glucose tolerance usually returns to normal in the majority of women with GDM,

\author{
ISSN 0857-1074 \\ Printed in the Philippines \\ Copyright (C) 2013 by the JAFES \\ Received March 24, 2013. Accepted April 23, 2013.
}

This paper was presented during the 2013 Annual Convention of the Philippine Society of Endocrinology and Metabolism, session on local research presentations, Philippine Research Initiatives on Diabetes and Endocrinology (PRIDE), EDSA Shangri-la Hotel, Manila, Philippines, March 22, 2013. over the long term, insulin secretion may deteriorate in relation to this chronic insulin resistance, leading to progressive hyperglycemia and type 2 diabetes. $^{7}$

Studies are varied in their estimates of risks of development of diabetes among women with previous GDM depending on ethnicity, cohort characteristics, diagnostic criteria used in the diagnosis of GDM and length of follow-up. 8 ,9 There were several reported maternal and pregnancy-related factors that are associated with the conversion to diabetes which include obesity, maternal age, previous history of GDM, family history of diabetes, parity, diagnosis of GDM early in pregnancy, high blood glucose levels at diagnosis, need for insulin treatment during pregnancy and an abnormal glucose tolerance test 2 months postpartum. ${ }^{8,10}$ The American Diabetes Association recommends that in the absence of obvious diabetes postpartum, women with GDM should be screened for diabetes 6-12 weeks postpartum using non-pregnant OGTT criteria and should be followed up 
with subsequent screening for the development of diabetes or prediabetes. If postpartum glucose levels are normal, reassessment of glycemia should be done at a minimum of 3-year intervals while women with impaired fasting glucose (IFG) or impaired glucose tolerance (IGT) should be tested annually. ${ }^{11}$ Despite these recommendations, postpartum testing rates are still suboptimal, ranging from $18 \%$ to $57 \% .^{12}$ Thus, some of these women with postpartum glucose abnormality may remain unidentified. Potential barriers to postpartum screening include lack of patient awareness of the increased risk of developing postpartum diabetes, and even if these women are aware of these risks, they may be unlikely to focus on their own health given the obligations of caring for a newborn. ${ }^{12}$

Data on the incidence of postpartum glucose intolerance in the Philippines is limited by poor follow-up of patients. A cross-sectional study looking at the prevalence of abnormal glucose tolerance and metabolic syndrome among Filipino women with previous GDM involving 65 patients has reported that as many as $32 \%$ were prediabetic and $17 \%$ were diabetic at postpartum (mean follow-up of 2.3 years). ${ }^{13}$ It is important to determine the true incidence of postpartum abnormal glucose tolerance to provide an estimate of the severity of the problem, to develop more effective strategies in the follow-up care of our GDM patients who could benefit from the early diagnosis and management of diabetes. The aim of this study is to determine the incidence of postpartum diabetes and glucose intolerance among women with GDM who delivered in our institution and to compare the risk factors present among these women.

\section{METHODOLOGY}

This is a prospective cohort study involving Filipino GDM patients aged 18 years and above who were admitted for delivery at the University of Santo Tomas Hospital (Private and Clinical Division) from October 2009 September 2012. Total enumeration was done in the recruitment of study subjects. Diagnosis of GDM was based on several criteria (Table 1) which includes the Carpenter and Coustan, World Health Organization criteria, and the latest recommendation by the American Diabetes Association and International Association of Diabetes and Pregnancy Study Group (IADPSG). Excluded from the study were those with pre-gestational diabetes (Type 1 or Type 2 diabetes), those with history of long term intake of drugs that can affect glucose metabolism such as steroids and beta adrenergic agonists and those with history of long term medical/surgical conditions that may affect glucose metabolism, such as post pancreatectomy, acromegaly and Cushing's syndrome. The study protocol was approved by the Institutional Review Board of UST Hospital. Written and informed consent were obtained from all participants upon recruitment.

\begin{tabular}{|c|c|c|c|}
\hline & $\begin{array}{c}\text { Carpenter and } \\
\text { Coustan } \\
(100 \mathrm{~g} \text { OGTT })\end{array}$ & $\begin{array}{c}\text { WHO }^{*} \\
\text { (75g OGTT) }\end{array}$ & $\begin{array}{c}\text { IADPSG^ } \\
\text { (75g OGTT) }\end{array}$ \\
\hline $\begin{array}{l}\text { Number of abnormal } \\
\text { values required for } \\
\text { GDM diagnosis }\end{array}$ & $\geq 2$ & $\geq 2$ & $\geq 1$ \\
\hline Fasting glucose (mg/dL) & 95 & 95 & 92 \\
\hline $1^{\text {st }}$ hour $(\mathrm{mg} / \mathrm{dL})$ & 180 & 180 & 180 \\
\hline $2^{\text {nd }}$ hour $(\mathrm{mg} / \mathrm{dL})$ & 155 & 155 & 153 \\
\hline $3^{\text {rd }}$ hour (mg/dL) & 140 & -- & - \\
\hline
\end{tabular}

\section{Data Collection and Outcome Measurements}

At the time of recruitment and at the visit performed postpartum, the following data were collected and recorded for each patient: age, height, and weight (prepregnancy weight was based on patient's recall), obstetric history including gestational age at diagnosis, parity, history of GDM, preeclampsia, macrosomia, stillbirth and/or abortion and family history of diabetes in the first degree relatives. Mode of therapy used (diet versus insulin) including the total dose of insulin used at term was recorded. Body mass index (BMI) was computed as weight in kilograms divided by height in meters squared. Based on BMI score, patients were classified as having normal weight $\left(\mathrm{BMI}=18.9-22.9 \mathrm{~kg} / \mathrm{m}^{2}\right)$, overweight (23$\left.24.9 \mathrm{~kg} / \mathrm{m}^{2}\right)$, obese I $\left(\mathrm{BMI}=25-29.9 \mathrm{~kg} / \mathrm{m}^{2}\right)$ and obese II (BMI of $>30 \mathrm{~kg} / \mathrm{m} 2) .{ }^{14}$ Birth outcomes were likewise recorded as follows: route of delivery (vaginal or cesarean section) and indication/s for cesarean section; presence of birth trauma and other complications; fetal birth weight, age of gestation, 1-minute and 5-minute APGAR scores; and the level of blood sugar in the immediate postpartum period.

The subjects were scheduled for a 75g OGTT at 6-12 weeks post partum. They were reminded through short messaging system (SMS) or calls at 4 weeks and on the day before their test. If the subject failed to follow up, reminders were sent every week through SMS messages. Investigators made every attempt to contact patients through cellular phone calls and residential numbers. Reminders were discontinued when patient did not answer telephone calls or when the patient expressed refusal to further participate in the study, or when a year had elapsed from the postpartum period.

Post-partum OGTT results were interpreted according to the American Diabetes Association criteria for diagnosis of diabetes. Diabetes mellitus type 2 (T2DM) is established when fasting plasma glucose is more than or equal to 126 $\mathrm{mg} / \mathrm{dL}$ or 2 hour post glucose loading level of more than or equal to $200 \mathrm{mg} / \mathrm{dL}$. Impaired fasting glucose is diagnosed when fasting plasma glucose is $\geq 100$ to 125 $\mathrm{mg} / \mathrm{dL}$ while IGT is diagnosed when $2 \mathrm{~h}$ post glucose loading level is $\geq 140$ to $199 \mathrm{mg} / \mathrm{dL}$. The main outcome measure was the development of diabetes mellitus, impaired fasting glucose and/or impaired glucose tolerance in the postpartum period. 


\section{Statistical Analysis}

The incidence of T2DM, IFG and/or IGT was computed by dividing the number of cases by the total number of GDM patients who were tested postpartum. Descriptive statistics such as mean and standard deviation were used to present data on demographic and clinical profile of the recruited subjects. Frequency distributions were used to present categorical data. Data were analyzed using STATA software version 10.1. One-way ANOVA was used to identify the presence of significant difference in the means of each group (Normal, IFG/IGT, and T2DM). Post-hoc test using Scheffe method of multiple comparisons was used to identify the group with significant difference from the normal. Two-sample test was used for proportions. Logistic regression was utilized for univariate analysis and model fitting to identify significant variables and estimate their effects on the respective outcomes. A p-value of $<0.05$ was considered to be of statistical significance.

\section{RESULTS}

There were 3,624 pregnant patients who delivered at the University of Santo Tomas Hospital (Private and Clinical Divisions) from October 2009 to September 2012. A total of $173(4.77 \%)$ subjects were diagnosed with GDM and met the inclusion and exclusion criteria. Table 2 shows the demographic profile of patients diagnosed with gestational diabetes mellitus. Patients had a mean age of $32.05 \pm 4.88$ years with a mean BMI of $23.77 \pm 9.3 \mathrm{~kg} / \mathrm{m}^{2}$ categorized as overweight based on the Asia-Pacific criteria for obesity. ${ }^{14}$ Majority of patients $(51.4 \%)$ had abnormal BMI, with as much as $8.1 \%$ classified under obese class II. Forty-five percent $(n=78)$ were primigravid. Of the multigravid patients, $27.2 \%(n=47)$ had previous history of abortion and $8.7 \%(\mathrm{n}=15)$ had prior preterm delivery.

Table 2. Demographic profile of patients with gestational diabetes mellitus

\begin{tabular}{|c|c|}
\hline$N=173$ & $N(\%)$ \\
\hline Age (years) (mean \pm sd) & $32.05 \pm 4.88$ \\
\hline (range) & $21-44$ \\
\hline Pregestational weight $(\mathrm{Kg})($ mean $\pm \mathrm{sd})$ & $57.36 \pm 9.30$ \\
\hline (range) & $40-90$ \\
\hline Pregestational BMI* $\left(\mathrm{Kg} / \mathrm{m}^{2}\right)$ (mean $\left.\pm \mathrm{sd}\right)$ & $23.77 \pm 3.89$ \\
\hline (range) & $16.63-37.66$ \\
\hline Underweight & $6(3.5)$ \\
\hline Normal & $78(45.1)$ \\
\hline Overweight & $40(23.1)$ \\
\hline Obese I & $35(20.2)$ \\
\hline Obese II & $14(8.1)$ \\
\hline Obstetrical History (mean \pm sd) & $1.95 \pm 1.10$ \\
\hline Gravida count (range) & $1-5$ \\
\hline Primigravida & $78(45.1)$ \\
\hline History of preterm delivery & $15(8.7)$ \\
\hline History of abortion & $47(27.2)$ \\
\hline History of macrosomia & $2(1.2 \%)$ \\
\hline History of pregnancy-induced hypertension & $9(5.2 \%)$ \\
\hline \multicolumn{2}{|l|}{ Past Medical History } \\
\hline History of hypertension & $8(4.6 \%)$ \\
\hline Family history of Diabetes & $104(60.1 \%)$ \\
\hline
\end{tabular}

Diagnosis of GDM was established using 100g OGTT in $78 \%$ of patients. Majority had abnormal $1^{\text {st }}$ and $2^{\text {nd }}$ hour glucose levels with mean values of $197.54 \pm 27.54 \mathrm{mg} / \mathrm{dL}$ and $180.79 \pm 34.69 \mathrm{mg} / \mathrm{dL}$, respectively. Of the 38 patients diagnosed with $75 \mathrm{~g}$ OGTT, $63.2 \%$ had abnormal $1^{\text {st }}$ hour post prandial levels. Fifty-five percent $(n=95)$ of patients were diagnosed during the third trimester of pregnancy (Figure 1). All patients were referred to St. Thomas Diabetes Center for education and proper nutrition. Fifty percent $(n=87)$ of patients required insulin therapy (range $=2-96$ units/day) to maintain normal glycemic targets.

Forty-eight percent of patients delivered via cesarean section (Table 3). The most common indication was repeat procedure secondary to previous history of cephalopelvic disproportion (49\%). Immediate postpartum capillary blood glucose monitoring showed normal mean pre-meals and post-meal levels of $97.92 \pm 14.11 \mathrm{mg} / \mathrm{dL}$ and $118.83 \pm$ $16.90 \mathrm{mg} / \mathrm{dL}$ respectively.

Table 3. Distribution of patients based on fetal and maternal outcomes

\begin{tabular}{|c|c|}
\hline Maternal Outcomes $(\mathrm{N}=173)$ & $\mathbf{N}(\%)$ \\
\hline \multicolumn{2}{|l|}{ Mode of delivery } \\
\hline Cesarean section & $83(48.0)$ \\
\hline \multicolumn{2}{|l|}{ Indication for Cesarean Section } \\
\hline Repeat procedure & $41(49)$ \\
\hline Arrest in cervical dilation & $20(24.1)$ \\
\hline Oligohydramnios & $7(8.4)$ \\
\hline Breech/transverse position & $3(3.6)$ \\
\hline Arrest in fetal descent & $3(3.6)$ \\
\hline Non-reassuring fetal pattern & $2(2.4)$ \\
\hline Severe Pre-eclampsia & $2(2.4)$ \\
\hline Placenta previa & $2(2.4)$ \\
\hline Preterm & $2(2.4)$ \\
\hline Congenital malformations & $1(1.2)$ \\
\hline \multicolumn{2}{|l|}{ Immediate postpartum glucose control } \\
\hline $\begin{array}{c}\text { Premeals }(\mathrm{mg} / \mathrm{dL}) \underset{\text { (range) }}{(\text { mean } \pm \mathrm{sd})} \\
\text { (ran }\end{array}$ & $\begin{array}{l}97.92 \pm 14.11 \\
70-154\end{array}$ \\
\hline Postmeals (mg/dL) (mean $\pm \mathrm{sd})$ & $118.83 \pm 16.90$ \\
\hline (range) & $80-185$ \\
\hline \multicolumn{2}{|l|}{ Fetal Outcomes } \\
\hline Term & $157(90.7)$ \\
\hline Preterm & $16(9.3)$ \\
\hline Birth weight $(\mathrm{Kg})$ & $2.72 \pm 0.56$ \\
\hline Macrosomia & $7(4.0)$ \\
\hline $\mathrm{AGA}^{*}$ & $151(87.3)$ \\
\hline LGA* & $14(8.1)$ \\
\hline $\mathrm{SGA}^{*}$ & $8(4.6)$ \\
\hline \multicolumn{2}{|l|}{$\mathrm{APGAR}^{\wedge}$ score } \\
\hline $\begin{array}{c}1 \text { min }(\text { mean } \pm s d) \\
\text { (range) }\end{array}$ & $\begin{array}{l}7.85 \pm 0.70 \\
4-9\end{array}$ \\
\hline $\begin{array}{c}5 \text { mins (mean } \pm s d) \\
\text { (range) }\end{array}$ & $\begin{array}{l}8.92 \pm 0.52 \\
5-9\end{array}$ \\
\hline Birth trauma & 0 \\
\hline Other complications & $16(9.2)$ \\
\hline Meconium aspiration & $5(2.9)$ \\
\hline Hypoglycemia & $4(2.3)$ \\
\hline Pneumonitis & $3(1.7)$ \\
\hline Sepsis & $2(1.2)$ \\
\hline Hydropnephrosis & $1(0.58)$ \\
\hline Inguinal hernia & $1(0.58)$ \\
\hline Neonatal Death & $4(2.3)$ \\
\hline
\end{tabular}

Ninety percent $(\mathrm{N}=157)$ delivered at full term with a mean birth weight of $2.72 \pm 0.56 \mathrm{~kg}$. Eight percent were large for gestational age, defined as birth weight above the $90^{\text {th }}$ percentile, of which 7 were macrosomic, defined as birth weight above $4 \mathrm{~kg}$. None of the babies delivered had birth trauma, however, $9.2 \%$ had other complications such as meconium aspiration, neonatal hypoglycemia and infection. There were 4 cases of fetal demise, 3 of which were delivered preterm. 


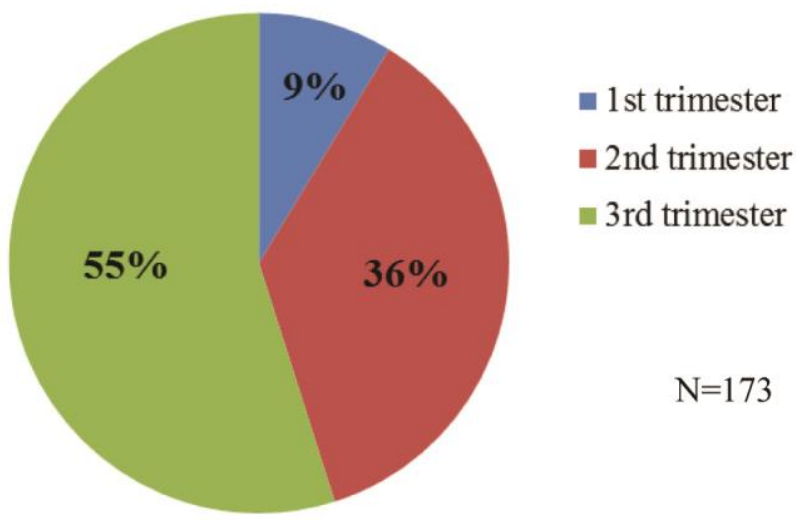

Figure 1. Distribution of women with GDM based on trimester of diagnosis.

\section{Incidence of Post-partum Glucose Intolerance}

Of the 173 patients, 49 did not follow-up despite constant reminders through SMS messages and calls. One hundred twenty-four patients had postpartum OGTT done and were analyzed in this study. The incidence of postpartum IFG, IGT and T2DM were 8.1, 19.4 and $7.3 \%$ respectively (Figure 2). Nine patients had combined IFG and IGT. Fiftyeight percent $(n=72)$ had normal postpartum OGTT results. Majority of the patients had OGTT done at 6-12 weeks post partum $(\mathrm{n}=100,80.6 \%)$. Sixteen $(12.9 \%)$ had follow-up OGTT at 13-24 weeks, while 8 (6.5\%) had the test at 28-48 weeks postpartum.

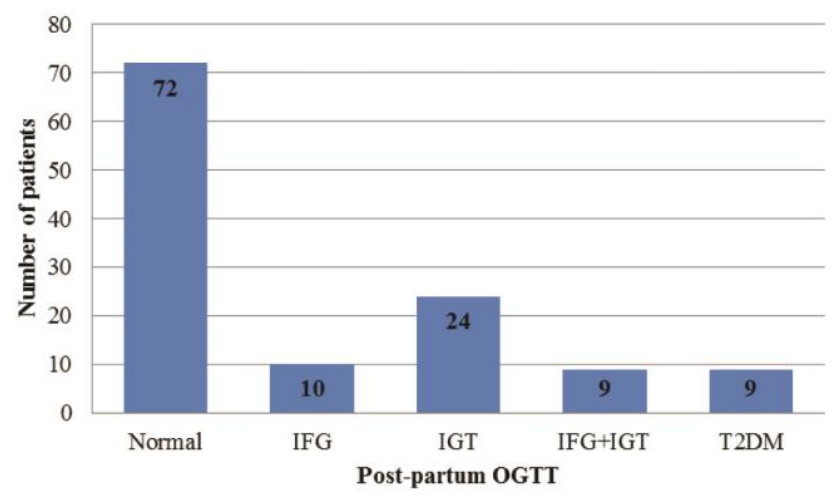

Figure 2. Distribution of women with GDM according to postpartum OGTT result

Based on their postpartum glycemic status, the subjects were divided into 3 groups (Normal, Prediabetes and Diabetes) and a comparison of their profile was done. Patients diagnosed with T2DM postpartum have higher mean age $(p=0.047)$, pre-gestational weight $(p=0.013)$ and BMI $(p=0.009)$. Mean values were higher for the prediabetes group when compared to normal but did not reach statistical significance (Table 4). For the maternal history, we found no significant differences between family history of diabetes, parity, prior history of GDM and macrosomia and history of pregnancy induced hypertension among the 3 groups (Table 5).
Table 4. Comparison of demographic characteristics of women according to postpartum glycemic status

\begin{tabular}{|c|c|c|c|}
\hline $\begin{array}{c}\text { Patient } \\
\text { Characteristics }\end{array}$ & $\begin{array}{c}\text { Normal } \\
\text { glucose } \\
\text { tolerance } \\
(n=72)\end{array}$ & $\begin{array}{l}\text { Pre-Diabetes } \\
\text { (IFG/IGT) } \\
(n=43)\end{array}$ & $\begin{array}{c}\text { Diabetes } \\
(n=9)\end{array}$ \\
\hline Age (years, mean \pm SD) & $31.14 \pm 4.86$ & $\begin{array}{l}32.74 \pm 4.35 \\
p=0.214\end{array}$ & $\begin{array}{l}34.78 \pm 5.24^{\star} \\
p=0.047\end{array}$ \\
\hline $\begin{array}{l}\text { Pre-gestational weight } \\
(\mathrm{Kg}, \mathrm{mean} \pm \mathrm{SD})\end{array}$ & $56.83 \pm 8.62$ & $\begin{array}{l}57.66 \pm 10.06 \\
p=0.675\end{array}$ & $\begin{array}{l}66.13 \pm 13.60 \\
p=0.013\end{array}$ \\
\hline $\mathrm{BMI}\left(\mathrm{Kg} / \mathrm{m}^{2}\right.$, mean $\left.\pm \mathrm{SD}\right)$ & $23.41 \pm 4.02$ & $\begin{array}{l}23.91 \pm 3.53 \\
p=0.736\end{array}$ & $\begin{array}{l}25.61 \pm 4.25^{\star} \\
p=0.009\end{array}$ \\
\hline
\end{tabular}

Table 5. Comparison of maternal history of women according to postpartum glycemic status

\begin{tabular}{|c|c|c|c|}
\hline & $\begin{array}{c}\text { Normal glucose } \\
\text { tolerance } \\
n=72 \\
N(\%) \\
\end{array}$ & $\begin{array}{c}\text { Pre-Diabetes } \\
\text { (IFG/IGT) } \\
n=43 \\
N(\%) \\
\end{array}$ & $\begin{array}{c}\text { Diabetes } \\
n=9 \\
N(\%)\end{array}$ \\
\hline $\begin{array}{l}\text { Family history of } \\
\text { DM }\end{array}$ & $52(72.2)$ & $\begin{array}{l}25(58.14) \\
p=0.12\end{array}$ & $\begin{array}{l}5(55.6) \\
p=0.069\end{array}$ \\
\hline $\begin{array}{l}\text { Gravida Count } \\
\text { (Mean } \pm \text { SD) }\end{array}$ & $1.52 \pm 0.93$ & $\begin{array}{l}1.86 \pm 0.99 \\
p=0.187\end{array}$ & $\begin{array}{l}1.25 \pm 0.5 \\
p=0.897\end{array}$ \\
\hline $\begin{array}{l}\text { History of preterm } \\
\text { delivery }\end{array}$ & $6(8.3)$ & $\begin{array}{l}3(6.98) \\
p=0.799\end{array}$ & $\begin{array}{l}0 \\
p=0.369\end{array}$ \\
\hline History of GDM & $5(6.9)$ & $\begin{array}{l}6(13.95) \\
p=0.213\end{array}$ & $\begin{array}{l}1(11.1) \\
p=0.649\end{array}$ \\
\hline $\begin{array}{l}\text { Macrosomia in } \\
\text { previous } \\
\text { pregnancies }\end{array}$ & $1(1.4)$ & $\begin{array}{l}1(2.3) \\
p=0.721\end{array}$ & $\begin{array}{l}0 \\
p=0.721\end{array}$ \\
\hline $\begin{array}{l}\text { Miscarriage or } \\
\text { stillbirth in previous } \\
\text { pregnancies }\end{array}$ & $12(16.7)$ & $\begin{array}{l}11(25.58) \\
p=0.249\end{array}$ & $\begin{array}{l}1(11.1) \\
p=0.666\end{array}$ \\
\hline $\begin{array}{l}\text { History of } \\
\text { pregnancy induced } \\
\text { hypertension }\end{array}$ & $5(6.9)$ & $\begin{array}{l}3(6.98) \\
p=0.987\end{array}$ & $\begin{array}{l}1(11.1) \\
p=0.649\end{array}$ \\
\hline
\end{tabular}

Women who had abnormal postpartum OGTT had higher $1^{\text {st }}$ hour and $2^{\text {nd }}$ hour values compared to normal (Table 6). Of the 97 patients diagnosed using the $100 \mathrm{~g}$ OGTT, those who developed T2DM had higher $3^{\text {rd }}$ hour glucose levels compared to the other 2 groups $(p=0.049)$. They were also noted to have higher number of positive results in the diagnostic OGTT during pregnancy $(p=0.024)$. Patients who had postpartum T2DM were diagnosed with GDM at an earlier age of gestation $(\mathrm{p}=0.004)$ (Table 7). A higher proportion of women with postpartum glucose intolerance were on insulin therapy, although this was not statistically significant. Those with T2DM had higher insulin doses during their pregnancy $(\mathrm{p}=0.042)$ which may be reflective of a greater state of insulin resistance.

Table 6. Comparison of OGTT values during pregnancy among women according to postpartum glycemic status

\begin{tabular}{|c|c|c|c|}
\hline & $\begin{array}{c}\text { Normal } \\
\text { glucose } \\
\text { tolerance } \\
(n=72)\end{array}$ & $\begin{array}{l}\text { Pre-Diabetes } \\
\text { (IFG/IGT) } \\
(n=43)\end{array}$ & $\begin{array}{l}\text { Diabetes } \\
(n=9)\end{array}$ \\
\hline $\begin{array}{l}\mathrm{FBS}(\mathrm{mg} / \mathrm{dL}, \\
\text { mean } \pm \mathrm{SD})\end{array}$ & $88.58 \pm 17.97$ & $\begin{array}{l}92.03 \pm 13.81 \\
p=0.682\end{array}$ & $\begin{array}{l}100.27 \pm 16.25 \\
p=0.0870\end{array}$ \\
\hline 1H OGTT & $186.9 \pm 24.18$ & $\begin{array}{l}198.4 \pm 26.15^{*} \\
p=0.0386\end{array}$ & $\begin{array}{l}211.35 \pm 29.7^{*} \\
p=0.0187\end{array}$ \\
\hline 2H OGTT & $164.1 \pm 23.71$ & $\begin{array}{l}183.8 \pm 32.17^{*} \\
p=0.009\end{array}$ & $\begin{array}{l}204.48 \pm 50.94^{*} \\
p=0.001\end{array}$ \\
\hline 3H OGTT^^ & $\begin{array}{l}134.7 \pm 29.36 \\
(n=53)\end{array}$ & $\begin{array}{l}134.0 \pm 33.0 \\
(n=35) p=0.9373\end{array}$ & $\begin{array}{l}163.11 \pm 60.83^{*} \\
(\mathrm{n}=9) \\
p=0.0490\end{array}$ \\
\hline $\begin{array}{l}\text { Total number } \\
\text { of positive } \\
\text { OGTT result }\end{array}$ & $2.1 \pm 0.81$ & $\begin{array}{l}2.37 \pm 0.76 \\
p=0.2195\end{array}$ & $\begin{array}{l}2.78 \pm 0.83^{*} \\
p=0.0243\end{array}$ \\
\hline
\end{tabular}


Table 7. Comparison of gestational age at diagnosis and mode of therapy among GDM patients according to postpartum glycemic status

\begin{tabular}{|c|c|c|c|}
\hline $\begin{array}{c}\text { Patient } \\
\text { Characteristics }\end{array}$ & $\begin{array}{c}\text { Normal glucose } \\
\text { tolerance } \\
(n=72) \\
N(\%)\end{array}$ & $\begin{array}{c}\text { Pre-Diabetes } \\
\text { (IFG/IGT) } \\
(n=43) \\
\text { N (\%) }\end{array}$ & $\begin{array}{c}\text { Diabetes } \\
(n=9) \\
N(\%)\end{array}$ \\
\hline $\begin{array}{l}\text { Trimester at } \\
\text { diagnosis } \\
(\text { mean } \pm S D)\end{array}$ & $2.58 \pm 0.60$ & $\begin{array}{l}2.37 \pm 0.76 \\
p=0.3036\end{array}$ & $\begin{array}{l}1.89 \pm 0.60^{\prime} \\
p=0.0037\end{array}$ \\
\hline Insulin therapy & $32(44.4)$ & $\begin{array}{l}22(51.16) \\
p=0.4822\end{array}$ & $\begin{array}{l}7(77.8) \\
p=0.0587\end{array}$ \\
\hline $\begin{array}{l}\text { Insulin Dose } \\
\text { (mean } \pm S D)\end{array}$ & $15.94 \pm 13.14$ & $\begin{array}{l}19.14 \pm 13.07 \\
p=0.6081\end{array}$ & $\begin{array}{l}26.71 \pm \\
19.81^{*} \\
p=0.0421\end{array}$ \\
\hline
\end{tabular}

The birth outcomes among the 3 groups were comparable (Table 8). Four neonatal mortalities were delivered from the group with normal and prediabetic postpartum OGTTs. Other factors such as preterm delivery and maternal hypertension may have affected this outcome. In Table 5, a higher proportion of patients in these 2 groups had gestational hypertension compared to those who had postpartum T2DM.

\begin{tabular}{|c|c|c|c|}
\hline $\begin{array}{c}\text { Patient } \\
\text { Characteristics }\end{array}$ & $\begin{array}{c}\text { Normal glucose } \\
\text { tolerance } \\
(n=72) \\
N(\%)\end{array}$ & $\begin{array}{l}\text { Pre-Diabetes } \\
\text { (IFG/IGT) } \\
(n=43) \\
\text { N (\%) }\end{array}$ & $\begin{array}{c}\text { Diabetes } \\
(n=9) \\
N(\%)\end{array}$ \\
\hline $\begin{array}{l}\text { Mode of Delivery } \\
\text { Cesarean }\end{array}$ & $35(48.61 \%)$ & $\begin{array}{l}20(46.51 \%) \\
p=0.8273\end{array}$ & $\begin{array}{l}3(33.3 \%) \\
p=0.3855\end{array}$ \\
\hline Preterm & $7(9.7 \%)$ & $\begin{array}{l}2(4.7 \%) \\
p=0.3342\end{array}$ & $\begin{array}{l}0 \\
p=0.3284\end{array}$ \\
\hline $\begin{array}{l}\text { Birthweight } \\
(\mathrm{Kg}, \text { mean } \pm \mathrm{SD})\end{array}$ & $2.97 \pm 0.52$ & $\begin{array}{l}3.07 \pm 0.49 \\
p=0.8103\end{array}$ & $\begin{array}{l}3.20 \pm \\
0.44 \\
p=0.6080\end{array}$ \\
\hline Macrosomia* & $3(4.2 \%)$ & $\begin{array}{l}3(6.98 \%) \\
p=0.5174\end{array}$ & $\begin{array}{l}0 \\
p=0.5309\end{array}$ \\
\hline $\begin{array}{l}\text { Large for } \\
\text { Gestational } \mathrm{Age}^{\wedge}\end{array}$ & $6(8.3 \%)$ & $\begin{array}{l}3(6.98 \%) \\
p=0.7985\end{array}$ & $\begin{array}{l}1(11.1 \%) \\
p=0.7777\end{array}$ \\
\hline $\begin{array}{l}\text { Presence of Birth } \\
\text { Trauma }\end{array}$ & 0 & 0 & 0 \\
\hline $\begin{array}{l}\text { Presence of } \\
\text { Neonatal } \\
\text { Complications }\end{array}$ & $7(9.7 \%)$ & $\begin{array}{l}4(9.3 \%) \\
p=0.9437\end{array}$ & $\begin{array}{l}1(11.1 \%) \\
p=0.8943\end{array}$ \\
\hline Neonatal mortality & $2(2.8 \%)$ & $\begin{array}{l}2(4.7 \%) \\
p=0.5922\end{array}$ & $\begin{array}{l}0 \\
p=0.6112\end{array}$ \\
\hline
\end{tabular}

Table 9 shows the comparison of the postpartum maternal factors. Women who had T2DM had significantly higher post-pregnancy weight $(p=0.006)$ and BMI $(p=0.004)$, although this may also be due to their higher prepregnancy weight and BMI. In terms of weight gained, there was no statistically significant difference between the groups.

\section{Risk factors and Postpartum OGTT Result}

A univariate analysis was done to identify variables that are associated with the result of OGTT on follow-up. A logistic regression model was fitted to provide better estimate of odds ratios. Factors significantly associated to having prediabetes postpartum are higher number of pregnancy and postpartum obesity (Table 10). Multigravid patients and those with $\mathrm{BMI} \geq 23 \mathrm{~kg} / \mathrm{m}^{2}$ are almost 3 times more likely to develop prediabetes compared to primigravid patients and those with normal BMI, respectively $(\mathrm{OR}=2.84 ; 95 \% \mathrm{CI} 1.20,6.70)$.

Table 9. Comparison of postpartum maternal factors among women with and without postpartum glucose intolerance

\begin{tabular}{|c|c|c|c|}
\hline $\begin{array}{c}\text { Patient } \\
\text { Characteristics }\end{array}$ & $\begin{array}{c}\text { Normal } \\
\text { glucose } \\
\text { tolerance } \\
(n=72)\end{array}$ & $\begin{array}{c}\text { Pre-Diabetes } \\
\text { (IFG/IGT) } \\
(n=43)\end{array}$ & $\begin{array}{l}\text { Diabetes } \\
(n=9)\end{array}$ \\
\hline $\begin{array}{l}\text { Postpartum weight } \\
\text { (Kg, mean } \pm S D)\end{array}$ & $57.75 \pm 8.48$ & $\begin{array}{l}59.7 \pm 8.21 \\
p=0.2298\end{array}$ & $\begin{array}{l}68.06 \pm 14.45^{*} \\
p=0.0063\end{array}$ \\
\hline $\begin{array}{l}\text { Postpartum BMI } \\
\left(\mathrm{Kg} / \mathrm{m}^{2}, \text { mean } \pm \mathrm{SD}\right)\end{array}$ & $23.97 \pm 3.73$ & $\begin{array}{l}24.68 \pm 3.09 \\
p=0.2621\end{array}$ & $\begin{array}{l}28.06 \pm 5.10^{*} \\
p=0.0038\end{array}$ \\
\hline $\begin{array}{l}\text { Postpartum wt gain } \\
(\mathrm{Kg}, \text { mean } \pm \mathrm{SD})\end{array}$ & $1.83 \pm 4.56$ & $\begin{array}{l}2.04 \pm 4.4 \\
p=0.9092\end{array}$ & $\begin{array}{l}1.93 \pm 3.72 \\
p=0.9848\end{array}$ \\
\hline
\end{tabular}

\begin{tabular}{|c|c|c|c|c|}
\hline Variables & $\begin{array}{l}\text { Unadjusted } \\
\text { OR (95\% Cl) }\end{array}$ & $p$-value & $\begin{array}{c}\text { Adjusted } \\
\text { OR } \\
(95 \% \mathrm{Cl})\end{array}$ & p-value \\
\hline Older Age & $\begin{array}{l}1.05 \\
(0.98,1.14)\end{array}$ & 0.186 & -- & -- \\
\hline $\begin{array}{l}\text { Pre-pregnant } \\
\text { Obesity }^{*}\end{array}$ & $\begin{array}{l}1.18 \\
(0.83,1.67)\end{array}$ & 0.359 & -- & -- \\
\hline Multigravida & $\begin{array}{l}2.51 \\
(0.09,5.77)\end{array}$ & 0.030 & $\begin{array}{l}2.84 \\
(1.20,6.70)\end{array}$ & 0.017 \\
\hline $\begin{array}{l}\text { Family History } \\
\text { of DM }\end{array}$ & $\begin{array}{l}0.58 \\
(0.27,1.26)\end{array}$ & 0.173 & -- & -- \\
\hline$\uparrow \mathrm{FBS}^{\wedge}$ & $\begin{array}{l}1.41 \\
(0.64,3.07)\end{array}$ & 0.391 & -- & -- \\
\hline$\uparrow 1 \mathrm{H} \mathrm{OGTT}{ }^{\wedge}$ & $\begin{array}{l}1.53 \\
(0.61,3.82)\end{array}$ & 0.361 & -- & -- \\
\hline$\uparrow 2 \mathrm{H} \mathrm{OGTT}{ }^{\wedge}$ & $\begin{array}{l}2.04 \\
(0.79,5.23)\end{array}$ & 0.138 & -- & -- \\
\hline Insulin Dose & $\begin{array}{l}1.18 \\
(0.85,1.68)\end{array}$ & 0.348 & -- & -- \\
\hline $\begin{array}{l}\text { Earlier Trimester } \\
\text { at Diagnosis }\end{array}$ & $\begin{array}{l}0.75 \\
(0.44,1.29)\end{array}$ & 0.296 & -- & -- \\
\hline $\begin{array}{l}\text { Postpartum } \\
\text { Obesity* }\end{array}$ & $\begin{array}{l}2.51 \\
(1.09,5.78)\end{array}$ & 0.030 & $\begin{array}{l}2.84 \\
(1.20,6.70)\end{array}$ & 0.017 \\
\hline
\end{tabular}

Factors associated with postpartum diabetes mellitus (Table 11) include obesity specifically a BMI $\geq 30 \mathrm{~kg} / \mathrm{m}^{2}$, use of insulin therapy especially those requiring higher insulin dosage during pregnancy and an earlier age of gestation at diagnosis of GDM. After adjustment, only earlier trimester at diagnosis and postpartum obesity remained significant. Diagnosis of GDM at an earlier trimester increases the odds of having postpartum T2DM 3-fold (OR=3.05; 95\% CI $1.02,9.18$ ) as compared to patients who had a GDM onset later. Also, if the BMI category falls under obese class II postpartum, the probability increases 115 times (95\% CI 3.96,3357.83; $\mathrm{P}=0.006$ ) compared to those with lower BMI. 


\begin{tabular}{|c|c|c|c|c|}
\hline Variables & $\begin{array}{l}\text { Unadjusted } \\
\text { OR }(95 \% \mathrm{Cl})\end{array}$ & p-value & $\begin{array}{l}\text { Adjusted OR } \\
(95 \% \mathrm{Cl})\end{array}$ & p-value \\
\hline Older Age & $\begin{array}{l}1.14 \\
(0.99,1.32)\end{array}$ & 0.074 & -- & -- \\
\hline $\begin{array}{l}\text { Pre-pregnant } \\
\text { Obesity* }\end{array}$ & $\begin{array}{l}5.89 \\
(1.26,27.6)\end{array}$ & 0.024 & $\begin{array}{l}0.05 \\
(0.01,1.97)\end{array}$ & 0.113 \\
\hline Multigravida & $\begin{array}{l}0.43 \\
(0.11,1.68)\end{array}$ & 0.22 & -- & -- \\
\hline $\begin{array}{l}\text { Family History } \\
\text { of DM }\end{array}$ & $\begin{array}{l}0.62 \\
(0.16,2.43)\end{array}$ & 0.490 & -- & -- \\
\hline$\uparrow \mathrm{FBS}^{\wedge}$ & $\begin{array}{l}2.98 \\
(0.74,11.9)\end{array}$ & 0.124 & -- & -- \\
\hline$\uparrow 1 \mathrm{H} \mathrm{OGTT}{ }^{\wedge}$ & $\begin{array}{l}0.90 \\
(0.17,4.71)\end{array}$ & 0.901 & -- & -- \\
\hline$\uparrow 2 \mathrm{H} \mathrm{OGTT}{ }^{\wedge}$ & $\begin{array}{l}1.26 \\
(0.14,11.0)\end{array}$ & 0.833 & -- & -- \\
\hline$\uparrow 3$ H OGTT^^ & $\begin{array}{l}1.43 \\
(0.36,5.69)\end{array}$ & 0.609 & -- & -- \\
\hline Insulin therapy & $\begin{array}{l}1.05 \\
(1.01,1.08)\end{array}$ & 0.016 & $\begin{array}{l}1.00 \\
(0.91,1.09)\end{array}$ & 0.952 \\
\hline $\begin{array}{l}\text { Higher Insulin } \\
\text { Dose }\end{array}$ & $\begin{array}{l}1.99 \\
(1.07,3.70)\end{array}$ & 0.031 & $\begin{array}{l}1.9 \\
(0.40,9.1)\end{array}$ & 0.422 \\
\hline $\begin{array}{l}\text { Earlier Trimester } \\
\text { at Diagnosis }\end{array}$ & $\begin{array}{l}3.17 \\
(1.25,8.06)\end{array}$ & 0.015 & $\begin{array}{l}3.05 \\
(1.02,9.18)\end{array}$ & 0.047 \\
\hline $\begin{array}{l}\text { Postpartum } \\
\text { Obesity* }\end{array}$ & $\begin{array}{l}12.34 \\
(2.7,56.5)\end{array}$ & 0.001 & $\begin{array}{l}115.3 \\
(3.96,3357.83)\end{array}$ & 0.006 \\
\hline $\begin{array}{l}{ }^{*} \text { Body Mass Index } \\
\text { ^Oral Glucose Tole } \\
\text { Statistical Tool: Lo }\end{array}$ & $30 \mathrm{~kg} / \mathrm{m}^{2}$ & ginc & & \\
\hline
\end{tabular}

\section{DISCUSSION}

The results of our study are consistent with data from other countries demonstrating the high incidence of postpartum glucose intolerance (T2DM and IFG/IGT) among women with previous history of GDM. We found the incidence of postpartum glucose intolerance to be high at $42 \%$ with $7.3 \%$ classified as having overt diabetes and $34.7 \%$ as having prediabetes. This incidence was slightly higher compared with our Asian counterparts. Jang et. al, reported the rates of postpartum T2DM and IGT in Korea to be $15.1 \%$ and $23.2 \%$ respectively $(38.3 \%$ ) at $6-8$ weeks postpartum. ${ }^{15}$ Similarly, a study by Ko et al., found that $35.8 \%$ of Chinese women had abnormal glucose tolerance post delivery ( $13.1 \%$ had diabetes and $22.7 \%$ had IGT). ${ }^{9} \mathrm{~A}$ study by Lin et. al., in Taiwan also showed incidence rates of diabetes and abnormal glucose tolerance to be $13.4 \%$ and $29.1 \%(42.5 \%)$ respectively. ${ }^{16}$

Several investigators in the past have identified factors associated with the conversion to diabetes in women with GDM. We have shown in our study that those who developed postpartum T2DM were older and had a higher pre-pregnancy weight and BMI. In terms of pregnancy related variables, the severity of hyperglycemia during pregnancy is a risk factor for future development of diabetes. Fasting hyperglycemia is generally regarded as a more significant metabolic abnormality than postprandial glucose level because it is thought to reflect insulin resistance rather than beta cell abnormality ${ }^{2}$ and fasting glucose levels from OGTT was predictive in the majority of the studies. ${ }^{8,16,17}$ Lin et. al., showed that fasting glucose value in the $100 \mathrm{~g}$ OGTT is the exclusive independent risk factor for DM or abnormal glucose tolerance. ${ }^{16}$ However, a particular threshold value predictive of development of diabetes was not identified in these studies since fasting glucose is usually adjusted as a continuous variable. In our study, fasting blood glucose levels were higher among those who developed postpartum glucose intolerance, however, this did not reach statistical significance (Table 6). The postprandial glucose levels in our subjects were significantly higher in the prediabetes and diabetes groups. Physiologically, the 1 hour blood glucose is thought to reflect $1^{\text {st }}$ phase insulin release which is believed to be deficient in GDM and T2DM patients and may explain the apparent better correlation. ${ }^{2}$ Those who had postpartum T2DM in our study were also noted to have a higher number of abnormal results in the diagnostic OGTT. The increasing number of positive values may reflect a more severe degree of glucose intolerance and a more severe degree of chronic insulin resistance and beta cell dysfunction in these patients. Similarly, Lin et. al., reported that presence of more than 3 abnormal values in the $100 \mathrm{~g}$ OGTT offers good diagnostic efficacy in predicting postpartum glucose intolerance. ${ }^{16}$ In this study, the cut-off point of 3 abnormal values in the $100 \mathrm{~g}$ OGTT achieved $86 \%$ sensitivity and $43 \%$ specificity in the prediction of postpartum diabetes or abnormal glucose tolerance. ${ }^{16}$

There have been inconsistent reports with regards to insulin use in pregnancy as a predictor for the long term development of T2DM. Several studies have shown that requirement of insulin therapy in GDM patients was the strongest predictor for the long term development of type 2 diabetes. ${ }^{2}$ Lobner et. al., also reported that compared with women who were diet controlled, women who received insulin during pregnancy had an almost 5-fold increased risk of developing type 2 diabetes. ${ }^{18}$ This relationship was significant in a dose dependent manner for an increase in insulin dose by 1 unit was associated with $5 \%$ risk increase for glucose intolerance after pregnancy. ${ }^{19}$ On the other hand, a study by Steinhart et. al., reported no statistically significant relationship with regards to insulin use during pregnancy and subsequent development of glucose intolerance. ${ }^{20}$ These differences may be due to the fact that insulin use during pregnancy depends on the clinician's judgments and patient preference/compliance and the use of other lifestyle interventions. Our study showed that those who were diagnosed with postpartum T2DM required higher insulin dose (mean= $26.71 \pm 19.81$ units/day) as compared to the other 2 groups. Also, the use of insulin therapy $(p=0.016)$ and higher insulin requirement $(p=0.031)$ during pregnancy are significant risk factors associated to postpartum T2DM.

Gestational age at diagnosis of GDM was also assessed as a risk factor for the subsequent development of diabetes in several studies. Jang et. al., found that for each week of increase in gestational age at diagnosis, there was a 0.99 
decrease in the odds of developing diabetes. ${ }^{15}$ In our data, diagnosis of GDM at an earlier trimester increases the odds of having postpartum T2DM 3-fold (OR=3.05; 95\% CI $1.02,9.18)$ as compared to patients who had a latter onset of GDM.

It has been studied that multiparity may hasten the conversion to diabetes because of the increased stress on beta cell function. ${ }^{19}$ In our study, multigravid and multiparous patients are 3 times $(\mathrm{OR}=2.84 ; 95 \% \mathrm{CI}$ $1.20,6.70)$ more likely to develop prediabetes compared to primigravid. We found no significant correlation between maternal age, family history of diabetes, prior history of GDM, history of pregnancy induced hypertension, macrosomia, stillbirth or abortion as well as in birth outcomes among the three groups.

Obesity is a well known risk factor for the development of T2DM. The central role of obesity has been shown to mediate a systemic inflammatory response with potential downstream insulin resistance and glucose dysregulation. ${ }^{16}$ In 1982, O'Sullivan indicated that there was a close relationship between obesity and development of T2DM after 10-16 years of follow-up in women with GDM. ${ }^{21}$ In another study, Pallardo et. al., found that, as compared with women with a pre-pregnancy BMI of $<27 \mathrm{~kg} / \mathrm{m} 2$, women with BMI of $>27 \mathrm{~kg} / \mathrm{m} 2$ had an 8 -fold increased risk of developing type 2 diabetes. ${ }^{22}$ These reports are consistent with our finding that the BMI of patients who had glucose intolerance postpartum was significantly higher compared with those who had normal glucose tolerance (T2DM=25.61 \pm 4.25 vs $\mathrm{IFG} / \mathrm{IGT}=23.91 \pm 3.53$ vs normal $\left.=23.41 \pm 4.02 \mathrm{~kg} / \mathrm{m}^{2}\right)$. The odds of having pre-diabetes postpartum increases 3 -fold $(\mathrm{OR}=2.84 ; 95 \% \mathrm{CI} 1.20,6.70)$ if the patient has abnormal BMI while the odds of having postpartum T2DM increases 115 times (95\% CI 3.96,3357.83; $\mathrm{p}=0.006)$ if the patient is classified under obese class II. Although postpartum BMI significantly correlated with the development of postpartum glucose intolerance, the amount of weight gained were similar in all groups. This may suggest that pre-pregnancy rather than postpartum BMI is a more important risk factor in the development of early postpartum diabetes or prediabetes in these women.

Although the American Diabetes Association has specific recommendations for postpartum glucose testing among women with GDM, testing is usually complicated by discontinuity of care after delivery and mothers underestimating their diabetes risk and stress to adapt to the challenge of caring for the young baby. ${ }^{8}$ Hence, usually less than $50 \%$ of women return for follow-up. ${ }^{17}$ In our study, $72 \%$ of women returned for follow-up testing. However, this could not be reflective of the true rate of follow-up in our population since these subjects were persistently reminded through text messages and/or calls beginning 4 weeks postpartum and the OGTT is funded by the researchers. It is important for us to note that despite these interventions, $28 \%$ of patients were still lost to follow-up. Majority of these patients stated that they are asymptomatic and that their priority is to care for their baby.

\section{CONCLUSION}

The incidence of postpartum diabetes and prediabetes (IFG/IGT) in our institution is $7.3 \%$ and $34.7 \%$ respectively. The risk of developing postpartum glucose intolerance increases with multigravid patients, with earlier onset of GDM during pregnancy and postpartum obesity. With this high incidence of conversion to diabetes and prediabetes (IFG/IGT), our study underscores the need for postpartum glucose testing in these women with GDM. The major limitation of this study is the high percentage of drop-outs at $28 \%$ which hindered us to determine the true incidence of postpartum diabetes and glucose intolerance and the identification of other risk factors and variables. Another limitation is the lack of standardized local criteria in the diagnosis of GDM. With the newer recommendations by the American Diabetes Association (2010) and the Philippine Obstetrics and Gynecology guidelines (2011), which have lower thresholds for GDM diagnosis, those who were not diagnosed with GDM using the WHO criteria prior to 2010, may have been labelled as having GDM using the newer criteria and should have been screened postpartum. We recommend that prospective studies be conducted using a larger population size to determine the effects of individual patient characteristics and risk factors predictive of the development of postpartum DM and glucose intolerance. Likewise, studies to identify variables that may improve patient compliance to postpartum glucose testing may be done. Clinicians should increase patient awareness of their risk of developing diabetes in the future and its associated microvascular and macrovascular complications. With the knowledge that obese patients are more likely to have diabetes postpartum, emphasis should be given on proper diet, exercise and weight loss. Furthermore, strategies to implement compliance to postpartum glucose testing must be formulated to increase rates of follow-up testing among these women.

\section{Acknowledgements}

We thank the following: Merck, Inc. through the Philippine Society of Endocrinology and Metabolism for the research grant awarded for this paper; consultants, fellows and staff of the USTH Section of Endocrinology and Metabolism for the support; Mr. Eriq Cruz for the statistical analysis of data; Residents of the Department of Medicine, Department of Obstetrics and Gynecology and Department of Pediatrics, Post-graduate interns and nursing staff of the Sta. Catalina Ward, OB Clinical Division ward and Delivery Room for the help, coordination and referrals of GDM patients. 


\section{References}

1. Friedman J, Kirwan J, Jing M, Presley L, Catalano P. Increased skeletal muscle tumor necrosis factor- $\alpha$ and impaired insulin signaling persist in obese women with Gestational Diabetes Mellitus 1 year postpartum. Diabetes. 2008; 57(3):606-613.

2. Lee AJ, Hiscock RJ, Wein PW, Walker SP, Permezel M. Gestational Diabetes Mellitus: Clinical predictors and long-term risk of developing Type 2 diabetes: A retrospective cohort study using survival analysis. Diabetes Care. 2007; 30(4):878-883.

3. Litonjua $\mathrm{AD}$, Waspadji $\mathrm{S}$, Pheng $\mathrm{CS}$, et al. AFES study group on diabetes in pregnancy preliminary data on prevalence. Phil J Internal Medicine. 1996; 34: 67-68.

4. Lim-Uy SW, Cunanan EC, Andag-Silva AA. Prevalence and risk factors of Gestational Diabetes Mellitus at the University of Santo Tomas Hospital. Phil J Internal Medicine. 2010; 48(1):24-31.

5. Buchanan T, Xiang A: Gestational Diabetes Mellitus. J Clin Invest. 2005; 115(3):485-491.

6. Rivas A, Gonzalez N, Gonzalez J. High frequency of diabetes in early post-partum assessment of women with gestational diabetes mellitus. Diabetes Metab Syndr Clin Res Rev. 2007; 1:159-165.

7. Metzger ED, Buchanan TA, Coustan DR, et al. American Diabetes Association: Summary and Recommendations of the Fifth International Workshop-Conference on Gestational Diabetes Mellitus. Diabetes Care. 2007; 30(Supp 2):s251-s260.

8. Kim C, Newton K, Knopp R. Gestational Diabetes and the incidence of Type 2 diabetes. Diabetes Care. 2002; 25(10):1862-1868.

9. Ko G, Chan J, Tsang L, Li C, Cockram C. Glucose intolerance and other cardiovascular risk factors in Chinese women with a history of Gestational Diabetes Mellitus. Aust N Z J Obstet Gynaecol. 1999; 39(4):478-483

10. Lauenborg J, Hansen T, Jensen DM, et al: Increasing incidence of diabetes after Gestational Diabetes. Diabetes Care. 2004; 27(5):11941199.

11. American Diabetes Association: Gestational Diabetes Mellitus. Diabetes Care. 2004; 27(Supp 1):S88-90.
12. Hunt K, Conway DL. Who returns for postpartum glucose screening following Gestational Diabetes Mellitus? Am J Obstet Gynecol 2008; 198(4): 404:e1-6.

13. Solimen D, Isip-Tan I. Abnormal glucose tolerance and metabolic syndrome among Filipino women with previous Gestational Diabetes Mellitus. 2006. Unpublished manuscript, University of the Philippines-Philippine General Hospital.

14. Kanazawa M, Yoshiike N, Osaka T, et al. Criteria and classification of obesity in Japan and Asia-Oceania. Asia Pacific Journal of Clinical Nutrition 2002; 11(s8): 732-737

15. Jang H, Yim C, Han K, et al. Gestational Diabetes mellitus in Korea: Prevalence and prediction of glucose intolerance at early postpartum. Diabetes Res Clin Pract. 2003; 61(2):117-124.

16. Lin $\mathrm{CH}$, Wen SF, Wu YH, Huang YY, Huang MJ: The postpartum metabolic outcome of women with previous Gestational Diabetes Mellitus. Chang Gung Med J. 2005; 28(11):794-800.

17. Ogonowski J, Miazgowski T. The prevalence of 6 weeks postpartum abnormal glucose tolerance in Caucasian women with gestational diabetes. Diabetes Res Clin Pract. 2009; 84(3):239-244.

18. Lobner K, Knoptf A, Baumgarten A, et al. Predictors of postpartum diabetes in women with Gestational Diabetes Mellitus. Diabetes. 2006; 55(3):792-797.

19. Krishnaveni G, Hill J, Veena S, et al. Gestational Diabetes and the incidence of diabetes in the 5 years following the index pregnancy in South Indian women. Diabetes Res Clin Pract. 2007; 78(3):398-404.

20. Steinhart JR, Sugarman JR, Connell FA: Gestational diabetes is a herald of NIDDM in Navajo women. High rate of abnormal glucose tolerance after GDM. Diabetes Care. 1997; 20(6):943-947.

21. O'Sullivan JB. Subsequent morbidity among GDM women: Carbohydrate metabolism in pregnancy and the newborn. New York, Churchill Livingstone 1984

22. Pallardo F, Herranz L, Garcia I: Early postpartum metabolic assessment in women with prior Gestational Diabetes. Diabetes Care. 1999; 22(7):1053-1058.

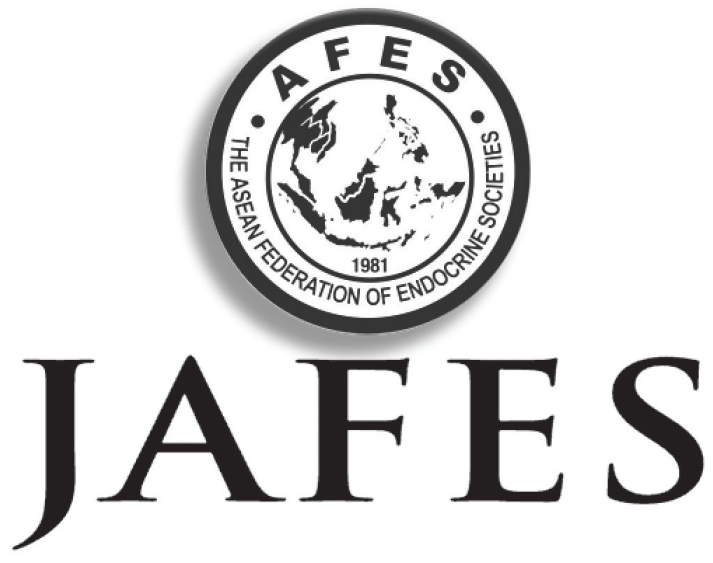

Experience the new JAFES.

Visit us at www.ASEAN-endocrinejournal.org. 